ARTICLE

\title{
Effects of Secondary Neutron Beam Generated in Radiotherapy on Electronic Medical Devices
}

\author{
Tomonori ISOBE ${ }^{1, *}$, Hiroaki KUMADA ${ }^{1}$, Kenta TAKADA ${ }^{1}$, Takayuki HASHIMOTO ${ }^{1}$, \\ Haruko HASHII ${ }^{1}$, Koichi SHIDA ${ }^{1}$, Kiichi TADANO ${ }^{1}$, Toshioh FUJIBUCHI ${ }^{1}$, \\ Masahiro HANMURA ${ }^{2}$, Hideyuki SAKURAI ${ }^{1}$ and Takeji SAKAE ${ }^{1}$ \\ ${ }^{1}$ Graduate School of Comprehensive Human Sciences, University of Tsukuba, \\ 1-1-1 Tennodai, Tsukuba, Ibaraki, 305-8575 Japan \\ ${ }^{2}$ Division of Radiation Oncology, Shizuoka Cancer Center Hospital, \\ 1007 Shimonagakubo, Nagaizumi-cho, Sunto-gun, Shizuoka, 411-8777 Japan
}

\begin{abstract}
In recent years, the administration of radiotherapy to patients bearing an implantable electronic device, such as a pacemaker, has been increasing. Although the effects of primary radiation on electronic devices have been studied, research on the effects of the secondary radiation beam is limited. Because semiconductors constitute the main parts of an electronic device, the effects of the secondary neutrons in radiation should be considered. We performed a Monte Carlo simulation of neutron generation during radiotherapy, and considered the influence of neutrons on the electronic medical devices. Monte Carlo simulations were performed using the Particle and Heavy Ion Transport code System (PHITS), developed by the Japan Atomic Energy Agency. Nuclear data was taken from ENDF/B-VII. We irradiated a water phantom with a $10 \mathrm{MV}$ X-ray beam and a $200 \mathrm{MeV}$ proton beam. Then, the neutron dose was evaluated by using PHITS. Moreover, we also measured the dose with a CR-39 dosimeter.

In the case of the $10 \mathrm{MV}$ X-ray beam, although fast neutrons $(>100 \mathrm{keV})$ were generated in the upstream part of the beam, thermal neutrons were scarcely generated. Inside the water phantom, which simulated a human body, the generation of fast neutrons decreased rapidly as a result of thermalization. In the case of the $200 \mathrm{MeV}$ proton beam, fast neutrons were distributed along the beam axis inside the water phantom, and were generated inside the water phantom. Thermal neutrons $(<0.5 \mathrm{eV})$ were uniformly distributed throughout the whole phantom.

In the evaluation of secondary neutrons generated during radiotherapy, we consider that using Monte Carlo calculations in addition to experimental measurements will be useful for clinical applications.
\end{abstract}

KEYWORDS: neutron, radiotherapy, electronic medical device, Monte Carlo simulation, PHITS, CR-39, dosimetry, proton, $X$-ray

\section{Introduction}

In recent years, the number of patients who undergo radiotherapy has increased; accordingly, radiation exposure not only to the patient but also to electronic medical devices implanted the human body has become an important topic in radiation biology. ${ }^{1,2)}$ Radiotherapy is already known to have a negative effect on electronic medical devices in the human body, owing to electromagnetic interference and other effects of ionizing radiation. ${ }^{3,4)}$

In the past, X-ray beams of $6 \mathrm{MV}$ or less have been used for radiotherapy. For these X-ray beam energies, the generation of neutrons through photonuclear reactions does not need to be taken into acount. ${ }^{5)}$ However, current radiotherapy uses X-ray beams with energy of $10 \mathrm{MV}$ or more. For this reason, neutron generation via photonuclear reactions cannot be ignored. Furthermore, the use of particle radiotherapy is also increasing. The effects of neutrons on medical devices such as pacemakers, implantable cardioverter defibrillators and ventriculoperitoneal shunts should be investigated. It is important to evaluate secondary neutrons,

*Corresponding author, E-mail: tiso@md.tsukuba.ac.jp

(C) 2011 Atomic Energy Society of Japan, All Rights Reserved. as well as to assess the risk and accuracy of radiotherapy. Moreover, radiation-induced cancer is also associated with secondary neutron exposure. ${ }^{6)}$

It has already been confirmed in previous research that electronic medical devices are damaged by direct irradiation with X-ray and proton beams. ${ }^{3,4,7)}$ The effects of scattered $\mathrm{X}$-rays on electronic medical devices have also been reported. ${ }^{8)}$ However, the effect of secondary neutrons generated in radiotherapy on such devices has not yet been clarified.

The purpose of the present research is to evaluate the effects of secondary neutrons on electronic medical devices through experimental measurements using CR-39 and through Monte Carlo simulations.

\section{Materials and Methods}

\section{Measurement with CR-39}

Figure 1 shows the experimental setup. A water phantom with 30-mm-thick polyethylene walls and dimensions of $24 \mathrm{~cm} \times 24 \mathrm{~cm} \times 24 \mathrm{~cm}$ was used to simulate the human body. The water phantom was placed on the treatment bed. 
(a)

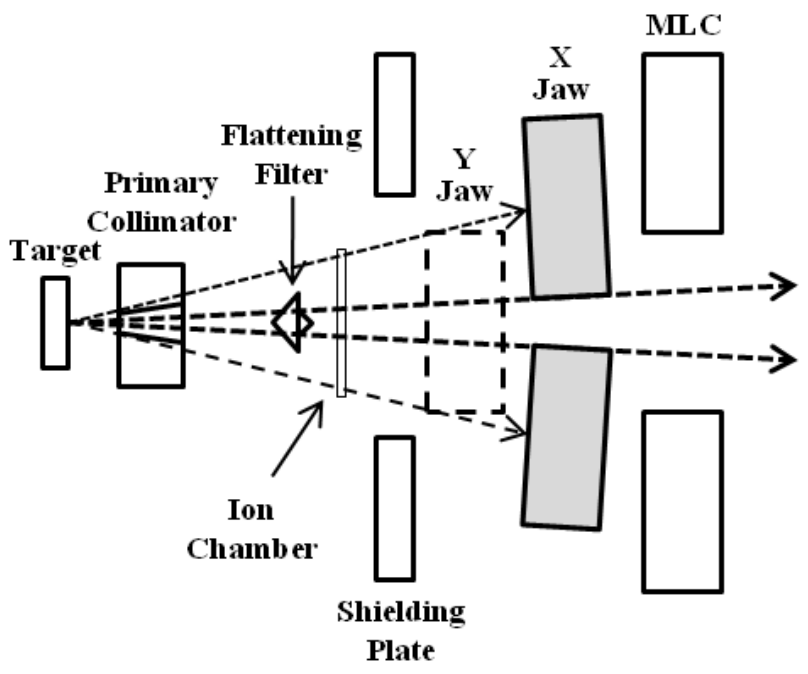

(b)

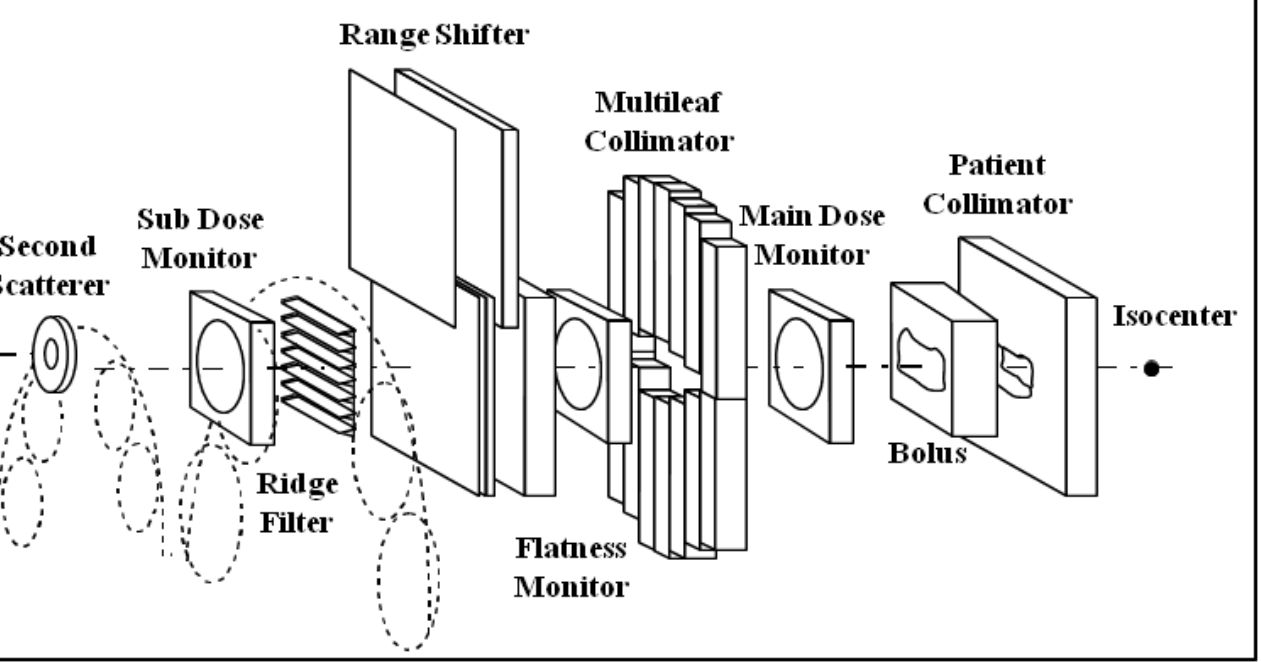

Fig. 1 Schematic of beam delivery system. (a) Cross-sectional image of Varian linear accelerator and (b) Side view of the proton beam line at the Proton Medical Research Center, University of Tsukuba.

For the measurements, a gantry angle of $90^{\circ}$ and X-ray and proton beam energies of $10 \mathrm{MV}$ and $200 \mathrm{MeV}$, respectively, were used. The X-ray beam was generated by a linear accelerator (Varian, Palo Alto, CA) (Fig. 1(a)). The proton beam was generated by a synchrotron (Hitachi, Tokyo, Japan) at the Proton Medical Research Center, University of Tsukuba. Figure 1(b) shows the setup of the proton beam line at our institution. The field size of the X-ray beam was set to $10 \mathrm{~cm} \times 10 \mathrm{~cm}$ with an isocenter depth of $12 \mathrm{~cm}$, which was at the center of the phantom. The field size of the proton beam was set to $10 \mathrm{~cm} \times 10 \mathrm{~cm}$; the proton beam was passed through a collimator made of brass. The spread-out Bragg peak (SOBP) was $6 \mathrm{~cm}$. The isocenter was at a depth of $17 \mathrm{~cm}$, which was the center of SOBP. We used CR-39 (Luxel badge; Nagase Landauer, Ibaraki, Japan) to measure the secondary neutron beam. CR-39 has been widely used as a personal neutron dosimeter that records the track of recoiled protons and $\alpha$ particles. ${ }^{9,10)}$ CR-39 was arranged at equal intervals on the phantom away from a beam axis and in parallel, in order to avoid direct radiation (Fig. 2). The measurement error in detection of neutrons with CR-39 de- pends on the number of neutrons. In order to minimize the measurement error, we predicted the dose of generated neutrons in advance, and then determined the exposure. The dosage was set to $300 \mathrm{~Gy}$ for the $10 \mathrm{MV}$ X-ray beam and $10 \mathrm{~Gy}$ for the $200 \mathrm{MeV}$ proton beam. Values measured with CR-39 were standardized to the track density per unit dose (Track $\cdot \mathrm{cm}^{-2} \cdot \mathrm{Gy}^{-1}$ ). The measurement energy range of CR-39 is below $0.5 \mathrm{eV}$ for thermal neutrons and more than $100 \mathrm{keV}$ for fast neutrons.

\section{Monte Carlo Simulation}

To estimate the dose of secondary neutrons, the conditions of the experiment using CR-39 were modeled in a numerical simulation, and then fluxes and the distribution of secondary radiation in the phantom were determined by Monte Carlo calculations. The Monte Carlo transport code PHITS (Japan Atomic Energy Agency: JAEA, Japan) was used to create a detailed model of an accelerator head, the geometry and composition of which were based on detailed technical drawings provided by the manufacturer. In performing transport calculations for photons, electrons, and neutrons 


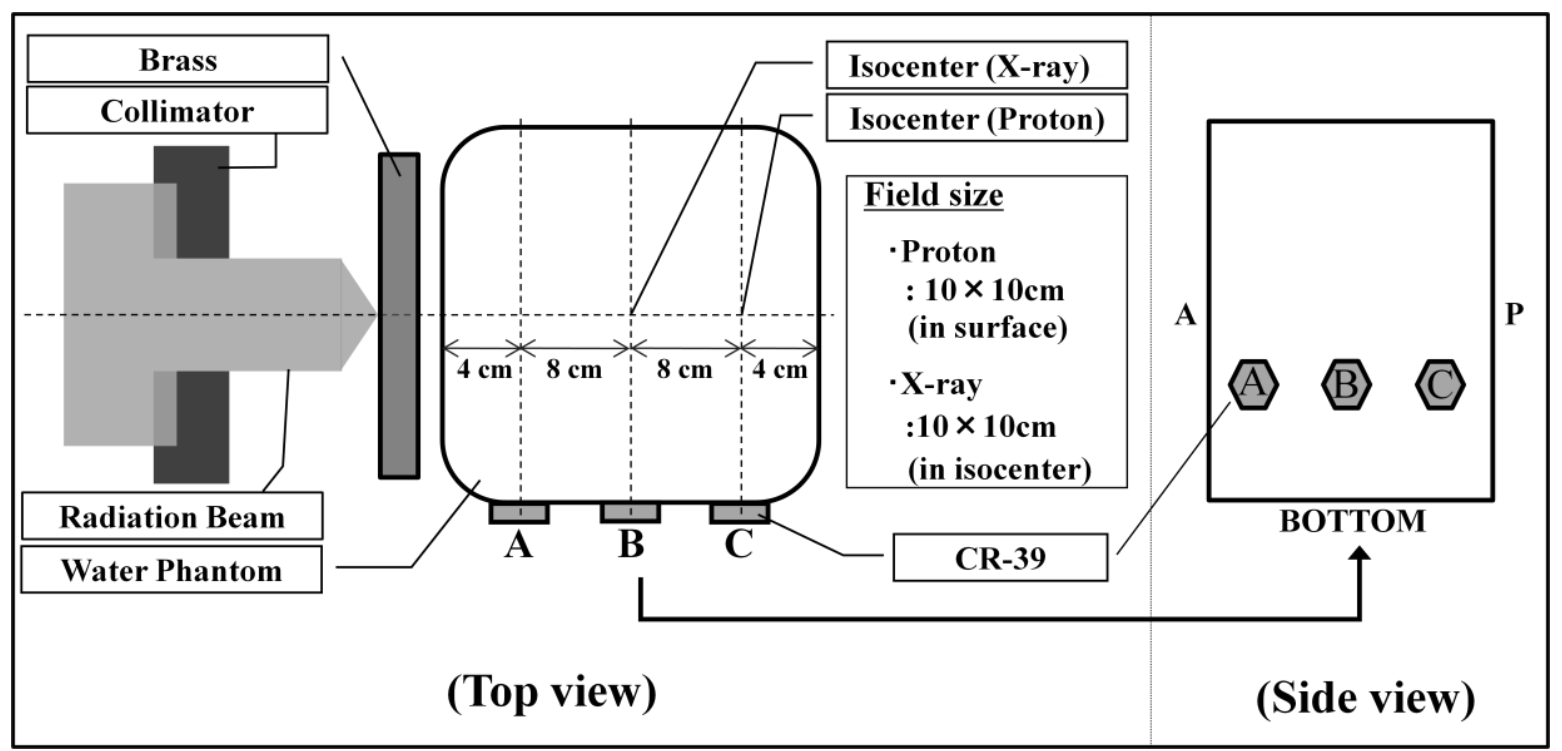

Fig. 2 Schematic of measurement setup (gantry angle: $90^{\circ}$ ).

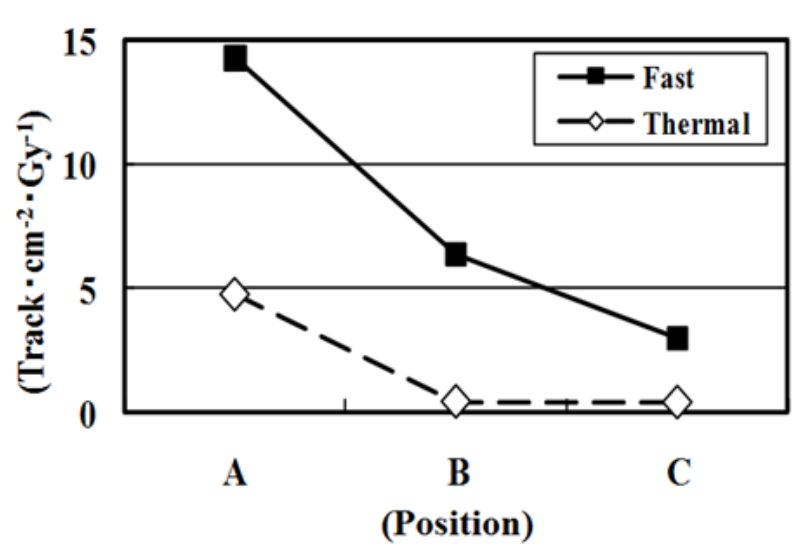

(a)

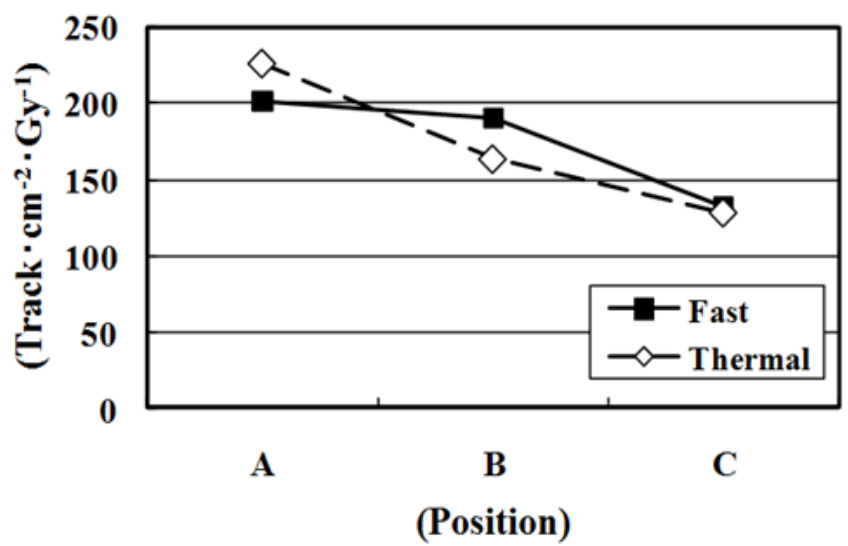

(b)

Fig. 3 Measured neutron dose (etch pit density) per unit dose (Tracks $\cdot \mathrm{cm}^{-2} \cdot \mathrm{Gy}^{-1}$ ) to a water phantom as a function of distance from the beam incidence plane for two radiotherapy beams: (a) $10 \mathrm{MV}$ X-ray beam and (b) $200 \mathrm{MeV}$ proton beam.

with energy below $20 \mathrm{MeV}$ in PHITS code, nuclear data is needed. Using PHITS, we can calculate not only the dose from proton beam exposure but also the transport of secondary neutrons. The nuclear data required for the Monte Carlo calculations was taken from ENDF/B-VII. For the $\mathrm{X}$-ray beam, we simulated the collision of $10^{8}$ electrons with a copper target. Then, the photonuclear reaction that occurred was analyzed. In the simulation of proton irradiation, the particle history number was set 6.5 million NPS, and both the therapeutic dose of protons and incidental dose of secondary radiation were determined. In the calculation, the energy of a neutron was set to coincide with the measurement energy range of CR-39 (thermal neutrons: $<0.5 \mathrm{eV}$; fast neutrons: > $100 \mathrm{keV}$ ).

\section{Results}

Figure 3 shows the distribution of the secondary neutron dose to the water phantom for various distances from the beam incidence plane. The neutron dose here is the number of etch pits, which is the number of cracks in CR-39 formed by recoiled protons and $\alpha$ particles. For the $10 \mathrm{MV} \mathrm{X}$-ray beam (Fig. 3(a)), fast neutrons decreased rapidly with increasing distance downstream from the beam. The dose of thermal neutrons was almost zero at the center of the water phantom. In the case of the proton beam (Fig. 3(b)), attenuation of the thermal neutrons and fast neutrons was slight, and they penetrated deep into the phantom. In comparison with the $10 \mathrm{MV}$ X-ray beam, the fast neutron dose resulting from the proton beam was 14 fold higher at position A, 30 fold higher at position $\mathrm{B}$ and 45 fold higher at position $\mathrm{C}$. In terms of thermal neutrons, the dose was 48 times higher at position A, 430 fold higher at position $\mathrm{B}$, and 366 fold higher at position $\mathrm{C}$.

Figure 4 shows the analysis results of the Monte Carlo simulation for the secondary neutron beam generated by irradiation with the $10 \mathrm{MV}$ X-ray beam. In the upstream part 


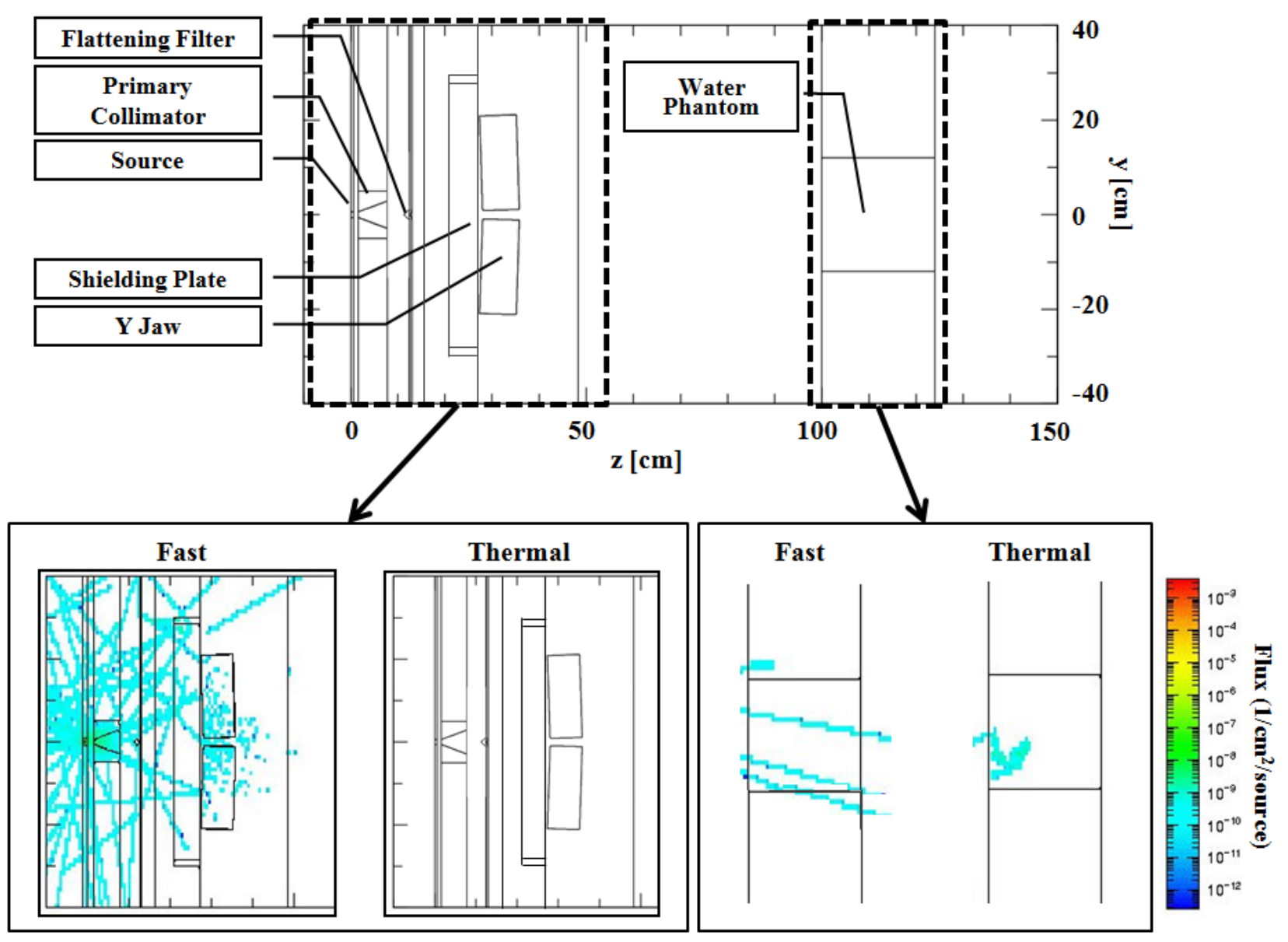

(a)

(b)

Fig. 4 Distribution of secondary neutrons generated by $10 \mathrm{MV}$ X-ray beam in Monte Carlo simulations: (a) upstream part of the beam and (b) interior of the water phantom.

of the beam (Fig. 4(a)), thermal neutrons were not generated, while fast neutrons were generated. As shown in Fig. 4(b), the tracks of the fast neutrons passed through the phantom. Thermal neutrons were also generated in the water phantom.

Figure 5 shows the analysis output of the Monte Carlo simulation of the secondary neutron beam generated when the water phantom was irradiated with the $200 \mathrm{MeV}$ proton beam. Fast neutrons were distributed along the beam axis. On the other hand, thermal neutrons were uniformly distributed throughout the entire phantom.

\section{Discussion}

In order to clarify the effects of the secondary neutrons generated by radiotherapy on implanted medical electronic devices, we evaluated the secondary neutrons in a comparison between CR-39 measurements and Monte Carlo simulations using PHITS. From the result of the Monte Carlo simulation, we found that thermal neutrons were clearly not generated in the upstream part of the beam during irradiation with the $10 \mathrm{MV}$ X-ray beam, whereas fast neutrons were generated. The fast neutrons were generated at the reaction of the upstream part of the beam and the primary beam. $^{11)}$
The fast neutrons generated in the upstream part of the beam reached the water phantom. In the case of the $10 \mathrm{MV}$ $\mathrm{X}$-ray beam, the distribution of fast neutrons inside the water phantom was not clear in the Monte Carlo results. This is a limitation of the PHITS code used in this research. We consider that the photonuclear reaction cannot be correctly calculated using PHITS. In the future, we plan to use the latest version of PHITS, which has a calculation function for the photonuclear reaction; then, we will compare the experimental results with the Monte Carlo simulation results. Using CR-39, we measured the secondary neutrons generated by the $10 \mathrm{MV}$ X-ray beam inside the water phantom, where the quantity of the fast neutrons decreased rapidly as they moved downstream. This is a phenomenon that accompanies thermalization of fast neutrons. Although we expected that the thermal neutrons would increase in the depth direction due to thermalization of fast neutrons, the thermal neutrons actually decreased. This finding can be explained by the fact that the part of CR-39 that detects thermal neutrons is also sensitive to fast neutrons. CR-39 consists of two neutron detectors, a polyethylene radiator and a boron nitride converter. Although CR-39 detects the thermal neutrons via the reaction with the boron nitride converter, fast neutrons also react with the boron nitride 


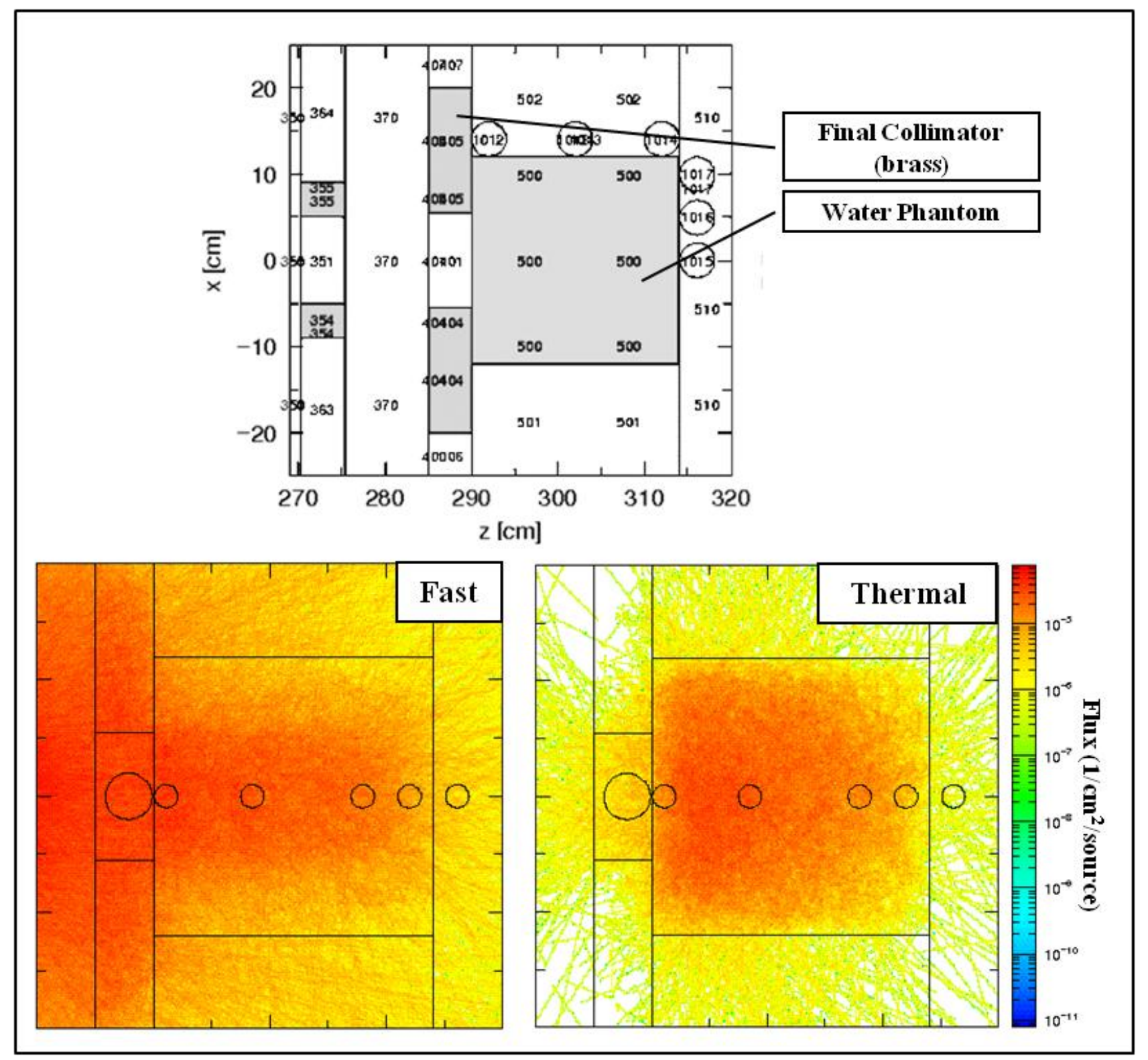

Fig. 5 Distribution of secondary neutrons generated by $200 \mathrm{MeV}$ proton beam inside the water phantom.

converter, and thus a fast neutron component is detected as thermal neutrons. In regard to this problem, we consider that thermal neutrons can be measured correctly by using activation of gold foils or CR-39 that is wrapped around its circumference with cadmium.

In the case of proton beam exposure, the Monte Carlo simulation inside a phantom was possible. The fast neutrons were distributed along the beam axis. On the other hand, the thermal neutrons were uniformly distributed over the whole phantom. Fast neutrons travel less than $10 \mathrm{~cm}$ in water because of thermalization. ${ }^{12)}$ Therefore, we expected that the value of fast neutrons measured with CR-39 at position B of Fig. 2 became zero. However, the value was hardly different from the value of the position of A. The distribution obtained from the Monte Carlo simulation (Fig. 5) agreed well with the measured distribution. These results show that the fast neutrons were generated inside the water phantom by the reaction between protons and water, and the neutrons then advanced into the phantom.

\section{Conclusions}

We evaluated the secondary neutrons generated during radiotherapy through measurements with CR-39 and through Monte Carlo simulations.

In the case of the $10 \mathrm{MV}$ X-ray beam, although fast neutrons were generated in the upstream part of the beam, thermal neutrons were scarcely generated. Inside the water phantom, which simulated the human body, the quantity of the fast neutrons decreased rapidly as a result of thermalization. In the case of the $200 \mathrm{MeV}$ proton beam, the fast neutrons were distributed along the beam axis inside the water phantom. Moreover, fast neutrons were generated inside the water phantom. The thermal neutrons were uniformly distributed throughout the whole phantom. 
Lastly, we will briefly discuss two areas that remain for future study. In CR-39, thermal neutrons cannot be measured correctly. Therefore, the first area of future research is to measure the thermal neutrons using another technique. In this study, the distribution inside the water phantom of fast neutrons generated by the $10 \mathrm{MV}$ X-ray beam could not be clearly simulated using PHITS. Therefore, the second area of future research is developing a more precise method for calculation of photonuclear reactions with PHITS.

Since the electron device consists of semiconductors, it is greatly influenced by a neutron. Therefore, in the evaluation of secondary neutrons generated during radiotherapy, we consider that using Monte Carlo calculations in addition to experimental measurements will be useful for clinical applications.

\section{Acknowledgement}

This work was supported in part by Nagase Landauer (Ibaraki, Japan).

\section{References}

1) R. M. Howell, N. E. Hertel, Z. Wang, J. Hutchinson, G. D. Fullerton, "Calculation of effective dose from measurements of secondary neutron spectra and scattered photon dose from dynamic MLC IMRT," Med. Phys., 33[2], 360-368 (2006).

2) G. Wilde, J. Sjostrand, "A clinical study of radiation cataract formation in adult life following gamma irradiation of the lens in early childhood," Br. J. ophthamol., 81[4], 261-266 (1997).

3) J. R. Marbach, M. R. Sontag, J. Van Dyk, A. B. Wolbarst, "Management of radiation oncology patients with implanted cardiac pacemakers: report of AAPM Task Group No. 34 American Association of Physicists in Medicine," Med. Phys. 21[1], 85-90 (1994).
4) C. W. Hurkmans, E. Scheepers, B. G. Springorum, H. Uiterwaal, "Influence of radiotherapy on the latest generation of implantable cardioverter-defibrillators," Int. J. Radiat. Onco.l Biol. Phys., 63[1], 282-289 (2005).

5) National Council on Radiation Protection and Measurements, "Neutron contamination from medical electron accelerators," NCRP Report 79 (1984).

6) U. Schneider, A. Lomax, N. Lombriser, "Comparative risk assessment of secondary cancer incidence after treatment of Hodgkin's disease with photon and proton radiation," Radiat. Res. 154[4], 382-388 (2000).

7) Y. Oshiro, S. Sugahara, M. Noma, M. Sato, Y. Sakakibara, T. Sakae, Y. Hayashi, H. Nakayama, K. Tsuboi, N. Fukumitsu, A. Kanemoto, T. Hashimoto, K. Tokuuye, "Proton beam therapy interference with implanted cardiac pacemakers," Int. J. Radiat. Oncol. Biol. Phys., 72[3], 723-727 (2008).

8) S. Kapa, L. Fong, C. R. Blackwell, M. G. Herman, P. J. Schomberg, D. L. Hayes, "Effects of scatter radiation on ICD and CRT function," Pacing Clin. Electrophysiol., 31[6], 727-732 (2008).

9) K. Oda, M. Ito, Y. Miyake, J. Yamamoto, T. Tsuruta, "Dose-equivalent response CR-39 track detector for personal neutron dosimetry," Nucl. Instr. Meth. Phys. Res., B61, 302-308 (1991).

10) R. J. Tanner, D. T. Bartlett, L. G. Hager, "Operational and dosimetric characteristics of etched-track neutron detectors in routine neutron radiation protection dosimetry," Radiat. Meas., 40[2-6], 549-559 (2005).

11) U. Titt, O. N. Vassiliev, F. Pönisch, S. F. Kry, R. Mohan, "Monte Carlo study of backscatter in a flattening filter free clinical accelerator," Med. Phys., 33[9], 3270-3273 (2006).

12) D. Shin, M. Yoon, J. Kwak, J. Shin, S. B. Lee, S. Y. Park, S. Park, D. Y. Kim, K. H. Cho, "Secondary neutron doses for several beam configurations for proton therapy." Int. J. Radiat. Onco.l Biol. Phys., 74[1], 260-265 (2009). 